\title{
Vigilancia de laboratorio de Streptococcus pneumoniae procedente de enfermedad invasora, Chile 2007-2012
}

\author{
M. Teresa Valenzuela, Mabel Seoane, Andrea Canals, Paola Pidal, Juan C. Hormazábal, \\ Pamela Araya, Solana Terrazas y Janepsy Díaz
}

\section{Laboratory surveillance of Streptococcus pneumoniae from invasive disease, Chile 2007-2012}

Background: 10-valent pneumococcal vaccine (PCV-10) was introduced in 2011 to the National Immunization Program in Chile. It was administered in 4 doses, but in 2012 it was modified to a 3 dose program. This article shows the results of the Laboratory Surveillance System for Streptococcus pneumoniae isolated of invasive disease from 2007 to 2012 and compares the incidence of invasive pneumococcal disease (IPD) by age groups in the prevaccinal (2007-2010) and postvaccinal period (2012). Methods: Descriptive study of S. pneumoniae surveillance in invasive diseases cases confirmed at the National Reference Laboratory of the Institute of Public Health of Chile from 2007 to 2012. Results: Global incidence of laboratory confirmed IPD cases decreased $27.8 \%$ from 2007 to 2012 and showed a lower risk for IPD in 2012 compared with 2007. Incidence in children aged 1 year or less decreased from 56.1 to 16.3 per 100,000 and from 42.0 to 19.9 per 100,000 in children aged 12 to 23 months in the same period. Highest decreases were observed in IPD cases caused by serotypes $4(100 \%), 19 \mathrm{~F}$ (93.3\%), 23F (90.9\%), $14(81.1 \%), 6 \mathrm{~B}(70 \%), 18 \mathrm{C}(58.3 \%)$ and $1(81.8 \%)$ in children aged 2 years or less. Conclusion: Surveillance System detects $S$. pneumoniae isolated from invasive diseases, contributing with information about laboratory confirmed IPD trends, prevalent serotypes and replacement effects. These results can be used as evidence in healthcare decision making for pneumococcal vaccines.

Key words: Pneumococcal vaccines, invasive pneumococcal disease, surveillance.

Palabras clave: Vacuna neumocóccica, enfermedad neumocóccica invasora, vigilancia.

\section{Introducción}

$\mathrm{D}$ espués de la introducción de la vacuna contra Haemophilus influenzae tipo b, la bacteria causal de enfermedades infecciosas invasoras que genera el mayor número de casos y muertes en los niños bajo 5 años de edad, es Streptococcus pneumoniae ${ }^{1}$, con una incidencia de enfermedad neumocóccica invasora (ENI) de 61/100.000 bajo 2 años y de 32/100.000 bajo 5 años ${ }^{2}$. Clínicamente la ENI se manifiesta como meningitis, bacteriemias, sepsis, artritis séptica o peritonitis ${ }^{3,4}$. Si bien la medición de carga de enfermedad neumocóccica a través de las infecciones invasoras nos conduce a una subestimación, tiene la ventaja que el segmento de la enfermedad medible se realiza mediante un método estándar, un cultivo de muestras estériles con aislamiento del agente causal, que a su vez permite disponer de información acerca de la importancia relativa de los serotipos causantes de ellas.

La primera vacuna desarrollada para prevenir las enfermedades causadas por este agente en lactantes contenía siete serotipos que cubrían entre 80 y $90 \%$ de los serotipos causantes de ENI en países desarrollados y $65 \%$ de los serotipos más prevalentes en países de América Latina ${ }^{6,7}$. La vacuna heptavalente demostró disminuir los casos de ENI, no sólo por los serotipos vacunales, sino que también por serotipos relacionados ${ }^{8}$. Las tasas de ENI en Estados Unidos de América disminuyeron $69 \%$ bajo dos años luego que en el año 2000 se introdujera esta vacuna ${ }^{9}$. A su vez, se estimó que con la introducción de una vacuna heptavalente se podría reducir los casos en $54 \%$ y las muertes en $53 \%$ y que, en la medida que se dispusiera de una vacuna con más serotipos, la reducción de la carga de enfermedad sería aun mayor ${ }^{2}$.

En el 2010, esta vacuna comenzó a ser remplazada por una de 13 serotipos y por otra de 10 serotipos $^{10}$. En Chile, esta última vacuna, que contiene los serotipos 1 , 4, 5, 6B, 7F, 9V, 14, 18C, 19F y $23 \mathrm{~F}$ de $S$. pneumoniae, fue incorporada al Programa Nacional de Inmunizaciones (PNI) para los lactantes que desde enero de 2011 fueran cumpliendo dos meses de edad, mediante un esquema de cuatro dosis (2-4-6-12 meses), el cual fue reemplazado en el año 2012 por un esquema de tres dosis (2-4-12 meses).

Para poder medir el impacto de la vacunación es necesario disponer de un sistema de vigilancia epidemiológica integral; uno de los componentes de ella es la vigilancia
Instituto de Salud Pública de Chile, Santiago, Chile. Dirección (MTVB) Departamento de Laboratorio Biomédico Nacional y de Referencia (MS, PP, JCH, PA). Departamento de Asuntos Científicos (AC, ST, JD).

Los autores de este manuscrito declaran no tener conflictos de interés.

Financiamiento: institucional.

Recibido: 25 de noviembre de 2013 Aceptado: 26 de octubre de 2014

Correspondencia a: María Teresa Valenzuela Bravo maritevalen@vtr.net 
a partir del laboratorio, mediante la confirmación de $S$. pneumoniae aislados de muestras estériles de casos con ENI, a través de métodos totalmente estandarizados. En Chile, y específicamente en la Región Metropolitana, gracias a un sistema de vigilancia de ENI, con base poblacional, se dispone de información de alta calidad respecto de la incidencia de ENI en los años previos a la introducción de la vacuna al programa. La incidencia medida de ENI en niños entre 0 meses y 35 meses fue de $30,7 / 100.000$ niños; la tasa más alta fue en lactantes bajo 5 meses con 61,0/100.000 niños ${ }^{11}$.

Actualmente en Chile la notificación de cepas de $S$. pneumoniae aisladas de ENI y su derivación al Laboratorio Nacional de Referencia del Instituto de Salud Pública de Chile (ISP) es obligatoria, tanto para laboratorios clínicos públicos y privados según lo establece el Decreto 158 de $2004^{12}$.

El objetivo de esta publicación es dar a conocer el resultado de la vigilancia de laboratorio de ENI en Chile desde el año 2007 al 2012 y comparar la incidencia según grupos de edades en un período prevacunal (2007-2010) con el postvacunal (2012).

\section{Material y Método}

Estudio descriptivo de la vigilancia de laboratorio de cepas de $S$. pneumoniae procedente de procesos invasores, confirmadas microbiológicamente y serotipificadas en el Laboratorio de Referencia (ISP), mediante el cual se calcula la incidencia de la enfermedad por grupos de edades. En este sentido, cada cepa de $S$. pneumoniae confirmada en el ISP corresponde a un caso de ENI con confirmación microbiológica.

El ISP es el Laboratorio Nacional y de Referencia para $S$. pneumoniae, y le corresponde confirmar, serotipificar y vigilar la susceptibilidad antimicrobiana de este agente recuperado de muestras de sitios estériles por los laboratorios clínicos públicos y privados del país ${ }^{12}$.

La confirmación microbiológica de las cepas se realiza a través de técnicas convencionales como solubilidad en bilis, tinción de Gram y susceptibilidad a la optoquina. La serotipificación capsular se realiza mediante la técnica de Quellung, según recomendaciones de la red SIREVAOPS/OMS ${ }^{13}$.

Para el análisis de las cepas de S. pneumoniae la base de datos fue depurada, de modo de asegurar que cada cepa corresponde a un caso de ENI confirmado microbiológicamente.

Dado que la vacuna neumocóccica conjugada 10-valente se introdujo en el programa a partir de enero de 2011, se compararon tres períodos en base a las diferentes etapas que ha tenido la implementación de la vacuna PCV-10 en Chile: período prevacunal, correspondiente a los años
2007 a 2010, período de transición, año 2011, y período postvacunal, año 2012, en cuanto a incidencias por año y por grupo de edad, según las proyecciones de población estimadas por el Instituto Nacional de Estadísticas (INE), promedio de casos de ENI bajo 2 años de edad, confirmados por año durante el período 2007-2010, producidos por cada serotipo (pre-vacunación), con los casos de ENI confirmados en el 2011 y 2012 , respectivamente.

\section{Análisis estadístico}

Se obtuvieron valores p para la tendencia y Odds Ratio (OR) con sus intervalos de confianza para comparar el riesgo de enfermar entre un año y otro, analizando la incidencia de un período sobre la incidencia del período de comparación (período anterior), considerando como sin riesgo (OR: 1) el año $2007 \mathrm{u}$ otro período definido. Se ajustaron modelos de Poisson para predecir el número de casos en lactantes bajo un año y niños de 12 a 23 meses para los años 2011 y 2012, en base a la información obtenida durante el período 2007-2010 y se comparó con los casos observados durante el año 2011 y el año 2012 .

Los datos se capturaron y procesaron en bases de datos. El análisis se realizó con el software EpiInfo ${ }^{\mathrm{TM}} 7$ y Stata 11.

\section{Componente ético}

Los datos analizados corresponden a la vigilancia epidemiológica obligatoria, definida mediante un decreto, D.S. N ${ }^{\circ} 158$ del 2004, sobre enfermedades de notificación obligatoria, en que los datos constituyen información pública, resguardando la confidencialidad de la identificación de las personas que se constituyen como casos.

\section{Resultados}

En el período 2007-2012 se confirmaron en el ISP 4.829 cepas de $S$. pneumoniae provenientes de igual número de casos de enfermedad invasora. El mayor número de cepas confirmadas se registró el 2009 (901), posteriormente el total de cepas confirmadas anuales descendió hasta 671, en el año 2012 (Figura 1).

\section{Incidencia}

La incidencia de ENI confirmadas por laboratorio experimentó un descenso a partir del año 2010 (valor p para tendencia: 0,01$)$ el cual se hace más evidente los años 2011 y 2012 (valor p para tendencia: < 0,0001). Al comparar el número de casos de ENI correspondiente al período 2007-2010 con el 2011 y el 2012 se observó una disminución significativa en el riesgo de enfermar con EI por $S$. pneumoniae en los años 2011 y 2012 (OR 2011 vs 2007-2010: 0,82 (IC 95\%: 0,75-0,89); OR 2012 vs 20072010: 0,76 (IC 95\%: 0,70-0,82)) (Figura 1). 
En la Tabla 1, se muestra la procedencia de las cepas $S$. pneumoniae en números absolutos y relativos por regiones de Chile. Se observó que en el período 2007-2012, el mayor porcentaje de cepas provino de la Región Metropolitana $(57,4 \%)$. Sin embargo, la vigilancia de laboratorio ha identificado casos de ENI en todas las regiones de Chile. Por otro lado, se observó que la procedencia de las cepas de $S$. pneumoniae durante todo el período fue mayor desde el sector público, abarcando alrededor de $80 \%$ del total de cepas estudiadas (Tabla 2).

\section{Incidencias por grupo etario}

La distribución de los cepas provenientes de ENI por grupo etario, permite observar que en el grupo de niños bajo un año de edad y de 12 a 23 meses existieron importantes cambios en el número absoluto de casos; bajo un año esta disminución fue de $41,7 \%$ entre 2007 y 2011 , y de $70,5 \%$ entre 2007 y 2012 . En el grupo de 12 a 23 meses ocurrió un fenómeno similar, disminuyendo en 15,4\% el número de cepas entre 2007 y 2011, y 51,9\% entre 2007 y 2012 (Figura 2). Se observó que los principales cambios en la incidencia se encuentran en el grupo bajo dos años. Bajo un año de edad, la incidencia específica disminuyó desde 56,1 a 16,3 por 100.000 niños. Esta disminución en el riesgo de enfermar de ENI comenzó a ser estadísticamente significativa a partir del año 2010 al compararlo con el período 2007-2009 (OR: 0,69; IC 95\%:
0,54-0,88) y continua los años 2011 (OR: 0,65; IC 95\%: $0,50-0,83)$ y 2012 (OR: 0,30; IC 95\%: 0,22-0,42). En el grupo de niños entre 12 y 23 meses, también se presentó un descenso en la incidencia de cepas provenientes de

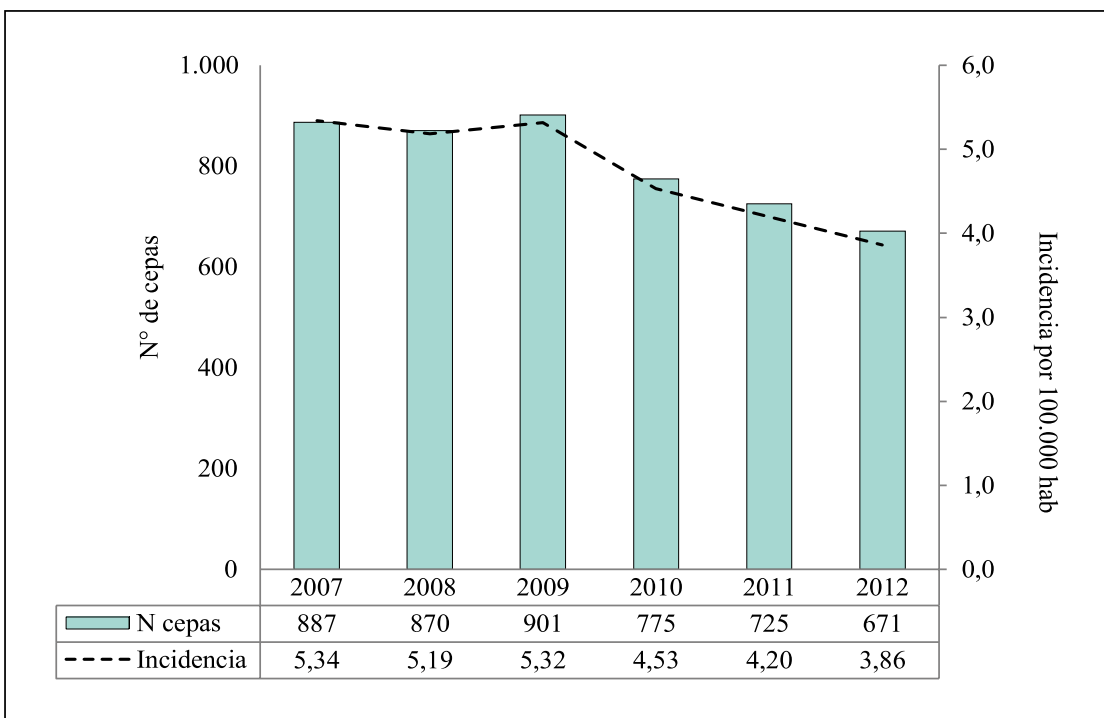

Figura 1. Número de cepas de S. pneumoniae provenientes de enfermedad invasora e incidencia de ENI por 100.000 habitantes, Chile, 2007-2012. Fuente: Laboratorio de Referencia de Meningitis Bacteriana. Departamento de Laboratorio Biomédico. ISP, 2013. ENI: enfermedad neumocóccicas invasora.

Tabla 1. Distribución de cepas confirmadas de S. pneumoniae de enfermedad invasora por año y región de procedencia. Chile, 2007-2012

\begin{tabular}{|c|c|c|c|c|c|c|c|c|c|c|c|c|c|c|}
\hline \multirow[t]{2}{*}{ Región } & \multicolumn{2}{|c|}{2007} & \multicolumn{2}{|c|}{2008} & \multicolumn{2}{|c|}{2009} & \multicolumn{2}{|c|}{2010} & \multicolumn{2}{|c|}{2011} & \multicolumn{2}{|c|}{2012} & \multicolumn{2}{|c|}{ Total } \\
\hline & n cepas & (\%) & n cepas & (\%) & n cepas & (\%) & n cepas & (\%) & n cepas & (\%) & n cepas & (\%) & n cepas & (\%) \\
\hline Tarapacá & 3 & 0,3 & 6 & 0,7 & 9 & 1,0 & 7 & 0,9 & 14 & 1,9 & 9 & 1,3 & 48 & 1,0 \\
\hline Antofagasta & 11 & 1,2 & 9 & 1,0 & 16 & 1,8 & 12 & 1,5 & 12 & 1,7 & 9 & 1,3 & 69 & 1,4 \\
\hline Atacama & 11 & 1,2 & 8 & 0,9 & 3 & 0,3 & 6 & 0,8 & 8 & 1,1 & 7 & 1,0 & 43 & 0,9 \\
\hline Coquimbo & 26 & 2,9 & 26 & 3,0 & 27 & 3,0 & 34 & 4,4 & 27 & 3,7 & 26 & 3,9 & 166 & 3,4 \\
\hline Valparaíso & 73 & 8,2 & 71 & 8,2 & 94 & 10,4 & 87 & 11,2 & 70 & 9,7 & 65 & 9,7 & 460 & 9,5 \\
\hline Libertador B.O'Higgins & 29 & 3,3 & 29 & 3,3 & 26 & 2,9 & 11 & 1,4 & 17 & 2,3 & 15 & 2,2 & 127 & 2,6 \\
\hline Maule & 22 & 2,5 & 22 & 2,5 & 15 & 1,7 & 10 & 1,3 & 14 & 1,9 & 15 & 2,2 & 98 & 2,0 \\
\hline Biobío & 78 & 8,8 & 67 & 7,7 & 64 & 7,1 & 54 & 7,0 & 76 & 10,5 & 66 & 9,8 & 405 & 8,4 \\
\hline Araucanía & 16 & 1,8 & 31 & 3,6 & 28 & 3,1 & 40 & 5,2 & 36 & 5,0 & 47 & 7,0 & 198 & 4,1 \\
\hline Los Lagos & 54 & 6,1 & 57 & 6,6 & 68 & 7,5 & 68 & 8,8 & 47 & 6,5 & 29 & 4,3 & 323 & 6,7 \\
\hline Aisén & 2 & 0,2 & 3 & 0,3 & 1 & 0,1 & 1 & 0,1 & 1 & 0,1 & 6 & 0,9 & 14 & 0,3 \\
\hline Magallanes & 0 & 0,0 & 0 & 0,0 & 1 & 0,1 & 3 & 0,4 & 1 & 0,1 & 0 & 0,0 & 5 & 0,1 \\
\hline Metropolitana & 549 & 61,9 & 527 & 60,6 & 526 & 58,4 & 426 & 55,0 & 389 & 53,7 & 356 & 53,1 & 2.773 & 57,4 \\
\hline Los Ríos & 10 & 1,1 & 10 & 1,1 & 21 & 2,3 & 15 & 1,9 & 12 & 1,7 & 19 & 2,8 & 87 & 1,8 \\
\hline Arica y Parinacota & 3 & 0,3 & 4 & 0,5 & 2 & 0,2 & 1 & 0,1 & 1 & 0,1 & 2 & 0,3 & 13 & 0,3 \\
\hline Total & 887 & 100 & 870 & 100 & 901 & 100 & 775 & 100 & 725 & 100 & 671 & 100 & 4.829 & 100 \\
\hline
\end{tabular}




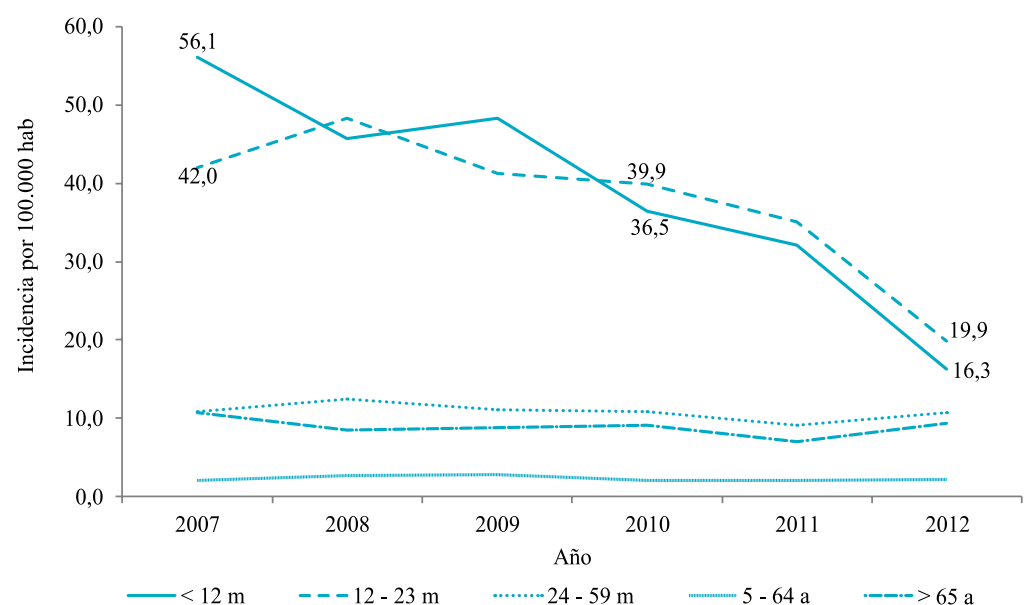

\begin{tabular}{|c|c|c|c|c|c|c|c|c|}
\hline \multirow{2}{*}{$\begin{array}{l}\text { Grupos } \\
\text { de edad }\end{array}$} & \multicolumn{6}{|c|}{ Número de cepas confirmadas por año (n) } & \multirow{2}{*}{$\begin{array}{c}2011 \\
\text { OR (IC 95\%) }{ }^{\dagger}\end{array}$} & \multirow{2}{*}{$\begin{array}{c}2012 \\
\text { OR (IC 95\%) })^{\ddagger}\end{array}$} \\
\hline & 2007 & 2008 & 2009 & 2010 & 2011 & 2012 & & \\
\hline $\mathrm{m}$ & 1 & 1 & 1 & 7 & 1 & 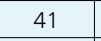 & $0,65(0,50-0,83)^{*}$ & $, 42)^{*}$ \\
\hline $12-23 m$ & 104 & 1 & 1 & & & & & \\
\hline $24-$ & & & & & & & & 0,9 \\
\hline$-64 a$ & 295 & 375 & 397 & 303 & 30 & 321 & $0,86(0,75-0,99)^{*}$ & $0,92(0$, \\
\hline$\geq 65 \mathrm{a}$ & 149 & 123 & 136 & 141 & 113 & 156 & $0,86(0,69-1,07)$ & $1,27(1,04-1,54)^{*}$ \\
\hline
\end{tabular}

†2007-2010 vs 2011 para cada grupo etario. ${ }^{\ddagger} 2007-2010$ vs 2012 para cada grupo etario. OR: Odds Ratio; IC: Intervalo de confianza; m: meses; a: años. Fuente: Laboratorio de Referencia de Meningitis Bacteriana. Departamento de Laboratorio Biomédico. ISP, 2013.

Figura 2. Incidencia de cepas provenientes de casos de enfermedad invasora confirmada microbiológicamente según grupo etario, Chile, 2007-2012.

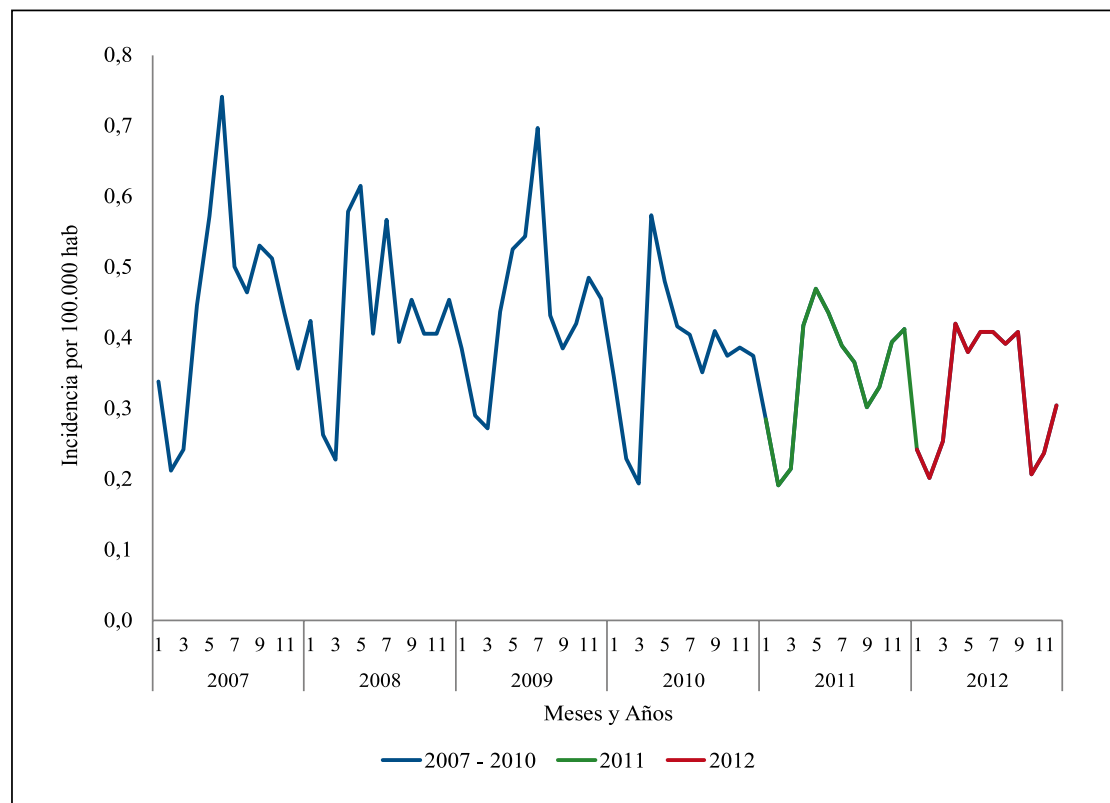

Figura 3. Incidencia de cepas de S. pneumoniae provenientes de casos de ENI confirmadas microbiológicamente por mes y año. Chile, 2007-2012. Fuente: Laboratorio de Referencia de Meningitis Bacteriana. Departamento de Laboratorio Biomédico. ISP, 2013.
Tabla 2. Distribución de cepas confirmadas de

S. pneumoniae provenientes de enfermedad invasora según procedencia, por año. Chile, 2007-2012

\begin{tabular}{lccccccc} 
Año & $\begin{array}{c}\mathbf{2 0 0 7} \\
\mathbf{( \% )}\end{array}$ & $\begin{array}{c}\mathbf{2 0 0 8} \\
\mathbf{( \% )}\end{array}$ & $\begin{array}{c}\mathbf{2 0 0 9} \\
\mathbf{( \% )}\end{array}$ & $\begin{array}{c}\mathbf{2 0 1 0} \\
\mathbf{( \% )}\end{array}$ & $\begin{array}{c}\mathbf{2 0 1 1} \\
\mathbf{( \% )}\end{array}$ & $\begin{array}{c}\mathbf{2 0 1 2} \\
\mathbf{( \% )}\end{array}$ & $\begin{array}{c}\text { Total } \\
\mathbf{( \% )}\end{array}$ \\
Público & 82,5 & 83,0 & 79,6 & 77,5 & 75,9 & 73,0 & 78,9 \\
Privado & 14,5 & 13,0 & 18,1 & 17,3 & 20,0 & 23,8 & 17,5 \\
Otros & 2,9 & 4,0 & 2,3 & 5,2 & 4,1 & 3,1 & 3,6 \\
Total & 100 & 100 & 100 & 100 & 100 & 100 & 100 \\
\hline
\end{tabular}

enfermedad invasora el año 2012 , desde 42,0 por 100.000 niños a 19,9 por 100.000 niños. Esto se traduce en una reducción del riesgo enfermar de ENI estadísticamente significativa comparado con el período prevacunal (OR: 0,44; IC 95\%: 0,32-0,59) (Figura 2).

\section{Estacionalidad}

Al analizar la incidencia de cepas provenientes de casos de enfermedad invasora confirmados microbiológicamente por mes y por año, se observó que durante los años 2007 a 2011 existe una estacionalidad marcada en los meses de invierno, lo cual no es tan pronunciado durante los años 2011 y 2012 (Figura 3).

\section{Serotipos}

Los mayores porcentajes de disminución en niños bajo 2 años de edad, al comparar el período 2007 2010 respecto de 2012, se observó en los casos de ENI producidos por serotipos $4(100 \%), 19 \mathrm{~F}(93,3 \%), 23 \mathrm{~F}$ $(90,9 \%), 14(81,1 \%), 6 \mathrm{~B}(70 \%), 18 \mathrm{C}(58,3 \%)$ y $1(81,8 \%)$ (Figura 4).

En la Tabla 3 se muestra la distribución de serotipos en casos de ENI confirmados microbiológicamente en niños bajo 12 meses de edad y 12-23 meses en los tres períodos: prevacunal, transición y postvacunal. En ocho de los serotipos contenidos en la vacuna 10 -valente, hubo una disminución de la importancia relativa para ambos por grupos de edades. Para el período 2007-2010, el más frecuente fue el serotipo 14 en ambos grupos etarios y en el 2012, el más frecuente fue el 19A bajo 12 meses de edad; sin embargo, en el grupo de 12 a 23 meses permanece el serotipo 14 como el más frecuente.

Mediante la aplicación de los modelos de Poisson ajustados para comparar el número de cepas recibidas (observado) desde casos bajo un año de edad y cepas de casos entre 12 y 23 meses de edad, versus lo esperado, entre los años 2007 y 2012 los resultados demostraron que hubo una reducción de cepas por sobre lo esperado del orden de 46,8\% y de 48,5\%, respectivamente (Figura 5). 


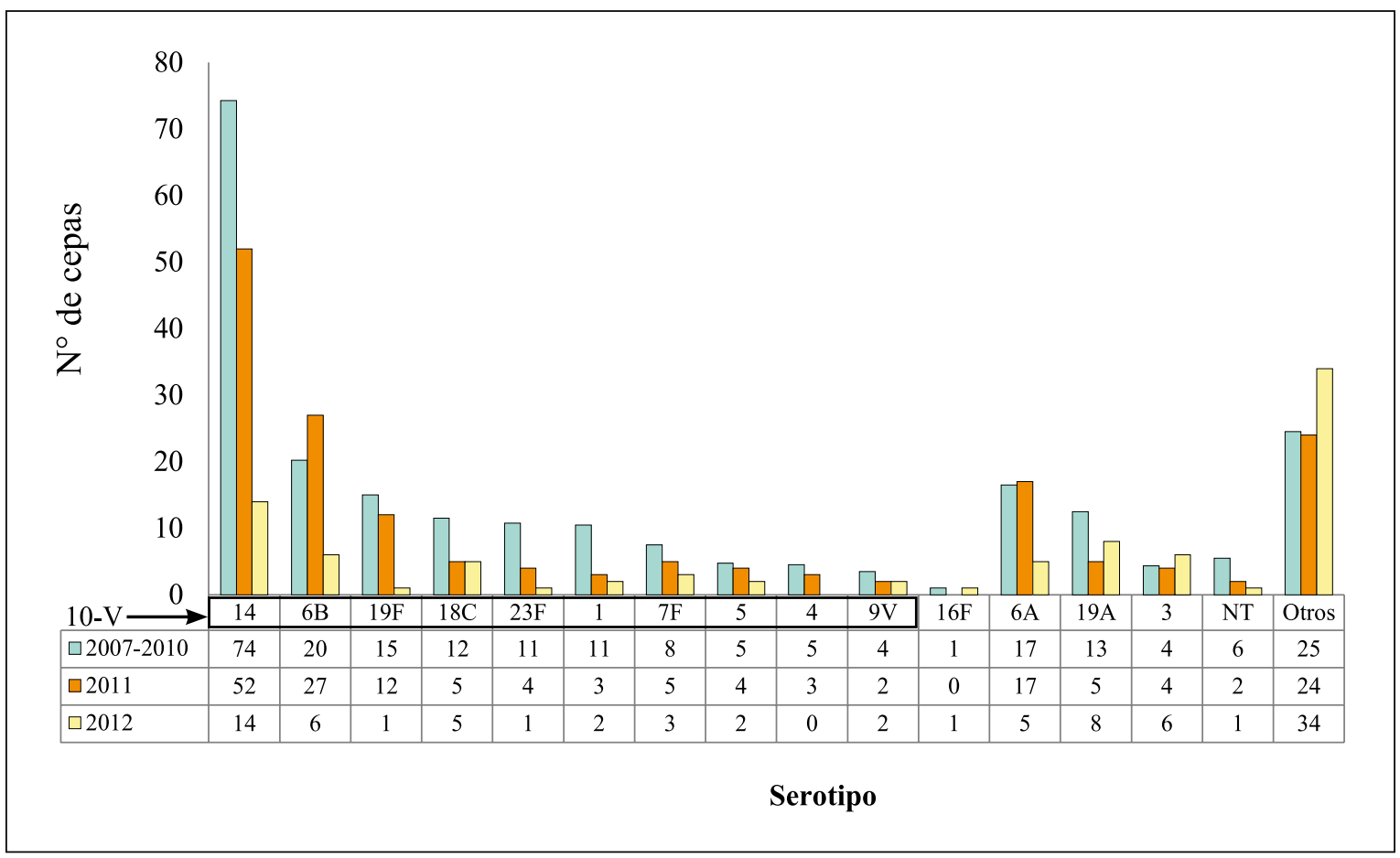

Figura 4. Comparación promedio de cepas de $S$. pneumoniae provenientes de casos de enfermedad invasora confirmados microbiológicamente en niños bajo 2 años de edad, según serotipo, entre período 20072010 y los años 2011 y 2012. Chile. Fuente: Laboratorio de Referencia de Meningitis Bacteriana. Departamento de Laboratorio Biomédico. ISP, 2013.

Tabla 3. Distribución de serotipos de cepas de S. pneumoniae provenientes de enfermedad invasora, por edad. Chile, 2007-2012

\begin{tabular}{|c|c|c|c|c|c|c|c|c|}
\hline \multirow{2}{*}{ Serotipo } & \multirow{2}{*}{$10 \mathrm{~V}$} & \multirow{2}{*}{$13 \mathrm{~V}$} & \multicolumn{3}{|c|}{$<12$ meses } & \multicolumn{3}{|c|}{$12-23$ meses } \\
\hline & & & $\begin{array}{c}2007-2010 \\
\%\end{array}$ & $\begin{array}{c}2011 \\
\%\end{array}$ & $\begin{array}{c}2012 \\
\%\end{array}$ & $\begin{array}{c}2007-2010 \\
\%\end{array}$ & $\begin{array}{c}2011 \\
\%\end{array}$ & $\begin{array}{c}2012 \\
\%\end{array}$ \\
\hline 1 & $x$ & $x$ & 4,9 & 1,2 & 2,4 & 4,4 & 2,3 & 2,0 \\
\hline 3 & & $x$ & 2,1 & 4,9 & 7,3 & 0,7 & 0,0 & 6,0 \\
\hline 4 & $x$ & $x$ & 1,7 & 0,0 & 0,0 & 2,3 & 3,4 & 0,0 \\
\hline 5 & $x$ & $x$ & 2,6 & 4,9 & 0,0 & 1,6 & 0,0 & 4,0 \\
\hline $6 \mathrm{~A}$ & & $x$ & 6,4 & 12,3 & 4,9 & 8,4 & 8,0 & 6,0 \\
\hline $6 \mathrm{~B}$ & $x$ & $x$ & 7,7 & 7,4 & 2,4 & 10,5 & 23,9 & 10,0 \\
\hline $7 F$ & $x$ & $x$ & 4,1 & 2,5 & 2,4 & 2,6 & 3,4 & 4,0 \\
\hline $9 \mathrm{~V}$ & $x$ & $x$ & 0,9 & 2,5 & 4,9 & 0,7 & 0,0 & 0,0 \\
\hline 14 & $x$ & $x$ & 30,7 & 30,9 & 7,3 & 36,1 & 30,7 & 22,0 \\
\hline $18 \mathrm{C}$ & $x$ & $x$ & 6,4 & 1,2 & 9,8 & 3,7 & 4,5 & 2,0 \\
\hline $19 \mathrm{~A}$ & & $x$ & 5,6 & 2,5 & 12,2 & 5,6 & 3,4 & 6,0 \\
\hline $19 \mathrm{~F}$ & $x$ & $x$ & 6,9 & 6,2 & 0,0 & 6,6 & 8,0 & 2,0 \\
\hline $23 \mathrm{~F}$ & $x$ & $x$ & 5,2 & 3,7 & 2,4 & 4,4 & 1,1 & 0,0 \\
\hline NT & & & 2,6 & 0,0 & 0,0 & 2,3 & 2,3 & 2,0 \\
\hline Otros & & & 12,2 & 19,8 & 43,9 & 9,8 & 9,1 & 34,0 \\
\hline Total & & & 100,0 & 100,0 & 100,0 & 100,0 & 100,0 & 100,0 \\
\hline$\%$ incluidos en vacuna 10 -valente ${ }^{\dagger}$ & & & 71,0 & 60,5 & 31,7 & 73,1 & 77,3 & 46,0 \\
\hline$\%$ incluidos en vacuna 13 -valente ${ }^{\dagger}$ & & & 85,2 & 80,2 & 56,1 & 87,8 & 88,6 & 64,0 \\
\hline
\end{tabular}




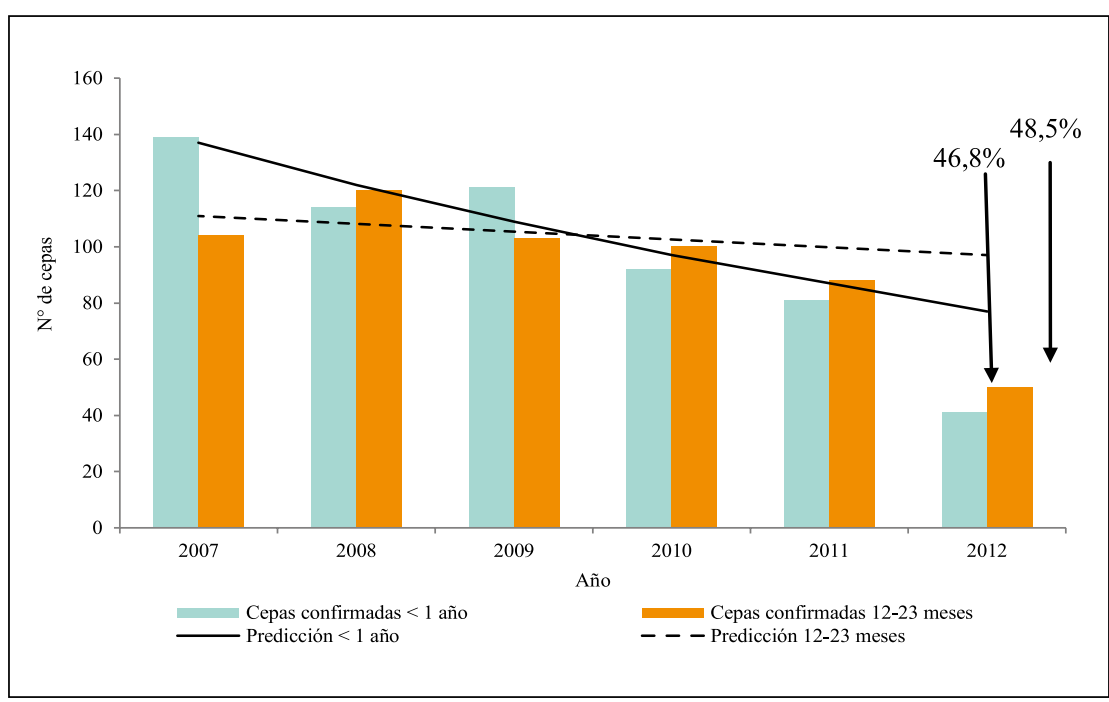

Figura 5. Número de cepas confirmadas esperadas y número de cepas confirmadas observadas de $S$. pneumoniae proveniente de enfermedad invasora para los años 2011 y 2012, por grupo de edad. Chile. Fuente: Laboratorio de Referencia de Meningitis Bacteriana. Departamento de Laboratorio Biomédico. ISP, 2013.

\begin{tabular}{|c|c|c|c|c|c|c|c|c|c|c|}
\hline \multirow{2}{*}{$\begin{array}{l}\text { Grupo } \\
\text { etario }\end{array}$} & \multicolumn{2}{|c|}{$1994-1999^{\dagger}$} & \multicolumn{2}{|c|}{$2000-2007$} & \multicolumn{2}{|c|}{$2007-2010^{\ddagger}$} & \multicolumn{2}{|c|}{2011} & \multicolumn{2}{|c|}{2012} \\
\hline & $\mathbf{n}$ & Tasa & $n$ & Tasa & $\mathbf{n}^{\ddagger}$ & Tasa & n & Tasa & $n$ & Tasa \\
\hline $0-5$ meses & 218 & 66,16 & 212 & 52,38 & 29 & 11,61 & 25 & 9,92 & 9 & 3,57 \\
\hline 6-35 meses & 413 & 24,86 & 787 & 38,41 & 155 & 20,73 & 104 & 13,80 & 71 & 9,42 \\
\hline Total & 631 & 31,70 & 999 & 40,72 & 184 & 24,60 & 129 & 17,12 & 80 & 10,61 \\
\hline
\end{tabular}

Fuente: CVD-Chile24. †Período entre 1 agosto de 1994 a 31 julio 1999. ${ }^{\ddagger}$ Media de casos ocurridos durante el período. Tasa de incidencia por 100.000 habitantes.

\section{Discusión}

La vigilancia de laboratorio de $S$. pneumoniae proveniente de enfermedad invasora en Chile ha permitido demostrar una reducción de la incidencia de cepas causantes de casos de enfermedad invasora confirmados por laboratorio en el grupo de niños bajo un año y en el grupo de 12 a 23 meses.

La tendencia a la disminución de la incidencia bajo un año se comenzó a observar antes del año 2010, previo al inicio de la vacunación universal del PNI; una posible causa sería que la vacuna neumocóccica 10-valente fue registrada por el Instituto de Salud Pública en el año 2009 e indicada en el sector privado de salud desde ese año.

La reducción de la carga de enfermedad neumocóccica ha sido demostrada en países donde se comenzó a usar la vacuna 7-valente revelando una importante disminución en la incidencia a partir del primer año de inclusión de la vacuna en los programas, en especial bajo un año de edad $^{9,14,15}$.

Pocos países han publicado su experiencia con la incorporación de la vacuna 10 -valente ${ }^{16,17}$. Hasta la fecha, en el contexto de los países de América Latina, Brasil quien incorporó la vacuna 10 -valente al programa, el año 2010 demostró que la reducción promedio anual de las tasas de hospitalización en niños de 2 meses de edad a 2 años, medida en el período de enero de 2005 a agosto de 2011, fue significativa, superior a $40 \%{ }^{16}$. También en Brasil, Domingues y cols., demostraron una efectividad de $83,8 \%$ para los serotipos incluidos en la vacuna y de $77,9 \%$ para los serotipos relacionados. De especial interés es el hallazgo de una efectividad de $82,2 \%$ contra el serotipo 19A, el que no se encuentra en la vacuna, siendo relacionado antigénicamente al $19 \mathrm{~F}^{18}$.

Otro estudio realizado en Argentina, Costa Rica y Panamá en niños, evaluó la eficacia vacunal contra neumonía adquirida en la comunidad probablemente bacteriana (NAC-B). Después de un seguimiento promedio de 23 meses la eficacia fue de 22\% (95\%IC 7,7-34,2) para NAC-B (p: 0,002) y $25,7 \%(8,4$ a 35,7$)$ para NAC consolidada según criterio OMS. La eficacia para otitis media aguda (OMA) causada por $S$. pneumoniae fue de $56,1 \%(95 \%$ IC $13,4-77,8)$ y de $67,1 \%(95 \%$ IC $17-86,9)$ para serotipos vacunales en análisis por protocolo, similar en intención de tratar y de $65 \%(95 \% \mathrm{IC} 11,1-86,9)$ para cualquier $\mathrm{ENI}^{19}$.

En Canadá, resultados obtenidos mediante un sistema de vigilancia centinela de la red de laboratorios, demostraron una reducción de la incidencia de ENI, tanto con el uso de la vacuna 7-valente como con la 10-valente, de 35 casos/100.00 personas-año y de 64 casos/100.000 personas-año, respectivamente ${ }^{17}$.

En cuanto a los serotipos prevalentes en Chile en el 2012 , en niños bajo un año, el 31,7 y 56,1\% estarían cubiertos por la vacuna 10 valente y la 13 valente, respectivamente. La diferencia entre ambas vacunas está dado por los serotipos 3 (7,3\%), 6 A (4,9\%) y 19 A (12,2\%), presentes en la vacuna 13 valente. De los serotipos cubiertos por la vacuna 10 -valente, el 14 experimentó la mayor disminución en su incidencia luego de introducida la vacuna. Otro hallazgo importante de destacar en esta vigilancia fue la marcada y sistemática estacionalidad de la incidencia de cepas de $S$. pneumoniae provenientes de enfermedad invasora, predominando en los meses de invierno. Este fenómeno se ha asociado al aumento en la incidencia de enfermedades respiratorias agudas que se presenta en forma de brotes epidémicos durante esos meses en Chile ${ }^{22}$.

Dado que en Chile, en la Región Metropolitana, varios años antes de la incorporación de la vacuna neumocóccica al Programa Nacional de Inmunizaciones, se realizaba una 
vigilancia activa y de alta calidad, a través de los servicios de Urgencia de los hospitales, liderada por el Centro de Vacunas en Desarrollo (CVD-Chile), se disponía de información sobre la incidencia de la ENI por grupos de edades $^{11,23}$. En la Tabla 4 se muestra el número de casos confirmados y la tasa de incidencia de ENI por 100.000 niños bajo tres años de edad, en los períodos 1994-1999 y $2000-2007^{24}$, y se compara con los hallazgos de esta vigilancia de laboratorio, correspondiente al período 2007-2012 en la Región Metropolitana. Las tasas correspondientes a niños de 0 a 5 meses se calcularon en base a la población de niños bajo un año y la tasa para el grupo de 6 a 35 meses en base a la población de niños bajo tres años, obtenidas de las proyecciones de población del INE. En el grupo de 0 a 5 meses se observó un descenso de $94,6 \%$ en la incidencia entre el período 1994-1999 y el año 2012, y de 64,0\% entre 2011 y 2012 . En el grupo de 6 a 35 meses la incidencia disminuyó en 62,1 y 31,7\% entre los mismos períodos.

Dentro de las limitaciones de este estudio se reconoce que la información analizada corresponde a cepas de $S$. pneumoniae provenientes de EI que fueron confirmadas por el ISP, lo que puede generar un sub-registro de casos a pesar del decreto que determina la notificación y confirmación de este agente en forma obligatoria ${ }^{12}$. Por tal motivo, en todo momento nos referimos a la incidencia de cepas provenientes de muestras estériles de casos de EI confirmados microbiológicamente, por el Laboratorio Nacional de Referencia.

Por otro lado, esta vigilancia no considera otras localizaciones ya que se enfoca en la ENI; por lo tanto, no se registran los casos de neumonía no bacteriemica o de OMA que podrían ser causadas por $S$. pneumoniae y evitados con la vacunación, como se ha demostrado en otros países ${ }^{25}$.

A pesar de las limitaciones de este estudio, queda demostrado que Chile mantiene un sistema de vigilancia que permite detectar cepas de $S$. pneumoniae aisladas de enfermedad invasora, lo que aporta información respecto de la tendencia de la ENI confirmada microbiológicamente y los serotipos prevalentes. A través de esta vigilancia se podrá estar alerta sobre el efecto de reemplazo de serotipos que se ha descrito en otros países luego de algunos años de iniciada la vacunación ${ }^{26}$.
Agradecimientos: A todo el personal del Instituto de Salud Pública involucrado en la Vigilancia de Laboratorio de Enfermedad Neumocóccica Invasora. A todos los integrantes de los equipos de salud del país que participan activamente de esta vigilancia.

\section{Resumen}

Introducción: La vacuna neumocóccica 10 valente fue incorporada al Programa Nacional de Inmunizaciones (PNI) desde enero de 2011 para lactantes mediante un esquema de cuatro dosis, y desde 2012, con un esquema de tres dosis. El objetivo de esta publicación es dar a conocer el resultado de la vigilancia de laboratorio de Streptococcus pneumoniae aislado de enfermedad invasora (ENI) desde el año 2007 al 2012 y comparar la incidencia de esta enfermedad según grupos de edades en un período prevacunal (2007-2010) con el postvacunal (2012). Materiales y Métodos: Estudio descriptivo de los resultados de la vigilancia de $S$. pneumoniae en los casos de ENI confirmados microbiológicamente en Chile, en el Laboratorio Biomédico Nacional de Referencia del Instituto de Salud Pública de Chile (ISP) durante los años 2007 a 2012. Resultados: La evolución de la incidencia global de $S$. pneumoniae en casos de ENI muestra un menor riesgo en los años estudiados (OR 2011 vs 20072010: 0,82 (IC 95\%: 0,75-0,89); OR 2012 vs 2007-2010: 0,76 (IC 95\%: 0,70-0,82)). En niños bajo un año de edad, la incidencia disminuyó desde 56,1 a 16,3 por 100.000 y en niños de 12 meses a 23 meses desde 42,0 a 19,9 por 100.000, en el mismo período. Los mayores porcentajes de disminución en los menores de 2 años se observaron en los casos de ENI producidos por los serotipos 4 (100\%), 19F (93,3\%), 23F (90,9\%), 14 (81,1\%), 6B (70\%), 18C $(58,3 \%)$ y $1(81,8 \%)$. Conclusión: El sistema de vigilancia permite detectar cepas de $S$. pneumoniae aisladas de enfermedad invasora en nuestro país, lo que aporta información respecto de la tendencia de la ENI confirmada microbiológicamente en Chile, los serotipos prevalentes y el posible efecto de reemplazo de ellos descrito en otros países, aportando a la autoridad de salud una herramienta adicional para la toma de decisiones respecto del tipo de vacuna a usar en el PNI con la mejor evidencia disponible.

\section{Referencias bibliográficas}

1.- O’Brien K L, Wolfson L J, Watt J P, Henkle E, Deloria-Knoll M, McCall N, et al. Burden of disease caused by Streptococcus pneumoniae in children younger than 5 years: global estimates. Lancet 2009; 374 (9693): 893-902.

2.- Valenzuela M T, O’Loughlin R, De La Hoz F, Gómez E, Constenla D, Sinha A, et al. The burden of pneumococcal disease among Latin American and Caribbean children: review of the evidence. Rev Panam Salud Publica 2009; 25 (3): 270-9.

3.- Levine M M, Lagos R, Levine O S, Heitmann I, Enríquez N, Pinto M E, et al. Epidemiology of invasive pneumococcal infections in infants and young children in Metropolitan Santiago, Chile, a newly industrializing country. Pediatr Infect
Dis J 1998; 17 (4): 287-93.

4.- Eskola J, Takala A K, Kela E, Pekkanen E, Kalliokoski R, Leinonen M. Epidemiology of invasive pneumococcal infections in children in Finland. JAMA 1992; 268 (23): 3323-7.

5.- Organización Panamericana de la Salud. Vigilancia de las neumonías y meningitis bacterianas en menores de 5 años: guía práctica. Washington, D C: OPS, (C) 2009; Publicación 
Científica y Técnica No. 633.

6.- Hausdorff W P, Bryant J, Paradiso P R, Siber G R. Which pneumococcal serogroups cause the most invasive disease: implications for conjugate vaccine formulation and use, part I. Clin Infect Dis 2000; 30 (1): 100-21.

7.- Robinson $\mathrm{K} \mathrm{A}$, Baughman W, Rothrock G, Barrett N L, Pass M, Lexau C, et al. Epidemiology of invasive Streptococcus pneumoniae infections in the United States, 1995-1998: Opportunities for prevention in the conjugate vaccine era. JAMA 2001; 285 (13): 1729-35.

8.- Whitney C G, Pilishvili T, Farley M M, Schaffner W, Craig A S, Lynfield R, et al. Effectiveness of seven-valent pneumococcal conjugate vaccine against invasive pneumococcal disease: a matched case-control study. Lancet 2006; 368 (9546): 1495-502.

9.- Whitney C G, Farley M M, Hadler J, Harrison L H, Bennett N M, Lynfield R, et al. Decline in invasive pneumococcal disease after the introduction of protein-polysaccharide conjugate vaccine. N Engl J Med 2003; 348 (18): 1737-46.

10.- WHO Publication. Pneumococcal vaccines WHO position paper -2012- recommendations. Vaccine 2012; 30 (32): 4717-8.

11.- Lagos R, Muñoz A, Valenzuela M T, Heitmann I, Levine M M. Population-based surveillance for hospitalized and ambulatory pediatric invasive pneumococcal disease in Santiago, Chile. Pediatr Infect Dis J 2002; 21 (12): 1115-23.

12.- Ministerio de Salud. Reglamento sobre Notificación de Enfermedades Transmisibles de Declaración Obligatoria. Diario Oficial 200510 Mayo; Decreto Supremo No 158/04.
13.- Organización Panamericana de la Salud. Procedimientos para el diagnóstico de Neumonías y Meningitis Bacterianas y la caracterización de cepas de Streptococcus pneumoniae y Haemophilus influenzae, SIREVA II. 2012.

14.- Ben-Shimol S, Greenberg D, Givon-Lavi N, Elias N, Glikman D, Rubinstein U, et al. Rapid reduction in invasive pneumococcal disease after introduction of PCV7 into the National Immunization Plan in Israel. Vaccine 2012; 30 (46): 6600-7.

15.- Fitzwater S P, Chandran A, Santosham M, Johnson H L. The worldwide impact of the seven-valent pneumococcal conjugate vaccine. Pediatr Infect Dis J 2012; 31 (5): 501-8.

16.- Afonso E T, Minamisava R, Bierrenbach A L, Escalante J J, Alencar A P, Domingues C M, et al. Effect of 10-valent pneumococcal vaccine on pneumonia among children, Brazil. Emerg Infect Dis 2013; 19 (4): 589-97.

17.- De Wals P, Lefebvre B, Defay F, Deceuninck G, Boulianne N. Invasive pneumococcal diseases in birth cohorts vaccinated with PCV-7 and/or PHiD-CV in the province of Quebec, Canada. Vaccine 2012; 30 (45): 6416-20.

18.- Domingues C M, Verani J R, Montenegro Renoiner E I, de Cunto Brandileone M C, Flannery B, de Oliveira L H, et al. Effectiveness of ten-valent pneumococcal conjugate vaccine against invasive pneumococcal disease in Brazil: a matched case-control study. Lancet Respir Med 2014; 2 (6): 464-71.

19.- Tregnaghi MW, Sáez-Llorens X, López P, Abate H, Smith E, Posleman A, et al. Efficacy of pneumococcal nontypable Haemophilus influenzae protein D conjugate vaccine (PHiD$\mathrm{CV}$ ) in young Latin American children: A double-blind randomized controlled trial. PLoS Med 2014; 11 (6): e1001657.

20.- Miller E, Andrews N J, Waight P A, Slack M P, George R C. Herd immunity and serotype replacement 4 years after seven-valent pneumococcal conjugate vaccination in England and Wales: an observational cohort study. Lancet Infect Dis 2011; 11 (10): 760-8.

21.- Kellner J D, Church D L, MacDonald J, Tyrrell G J, Scheifele D. Progress in the prevention of pneumococcal infection. CMAJ 2005; 173 (10): 1149-51.

22.- Instituto de Salud Pública de Chile. Ministerio de Salud. Vigilancia de Virus Respiratorios. 2013; Available at: http://www.ispch.cl/ virusrespiratorios. (accedido 31 de octubre de 2013).

23.- Lagos R, di Fabio J L, Moenne K, Muñoz M A, Wasserman S, de Quadros C. The use of chest $\mathrm{X}$-rays for surveillance of bacterial pneumonias in children in Latin America. Rev Panam Salud Publica 2003; 13 (5): 294-302.

24.- Lagos R, Muñoz A, San Martin O, Maldonado A, Hormazábal JC, Blackwelder WC, et al. Age -and serotype- specific pediatric invasive pneumococcal disease: insights from systematic surveillance in Santiago, Chile, 1994-2007. J Infect Dis 2008; 198 (12): 1809-17.

25.- Kellner J. Update on the success of the pneumococcal conjugate vaccine. Paediatr Child Health 2011; 16 (4): 233-40.

26.- Feikin D R, Kagucia E W, Loo J D, Link-Gelles R, Puhan M A, Cherian T, et al. Serotype-specific changes in invasive pneumococcal disease after pneumococcal conjugate vaccine introduction: a pooled analysis of multiple surveillance sites. PLoS Med 2013; 10 (9): ve1001517. 